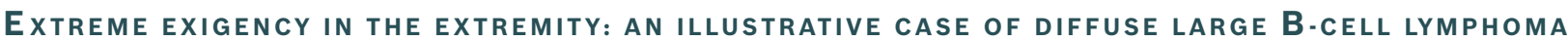

\section{OCCURRING IN THE ARM}

Singapore Med J 2018; 59(7): 397-398 https://doi.org/10.11622/smedj.2018092

Dear Sir,

Diffuse large B-cell lymphoma (DLBCL) continues to account for a major proportion of all non-Hodgkin's lymphoma subtypes. ${ }^{(1,2)}$ Although the outcomes have not been reported to be distinct, clinical presentations of extranodal DLBCL remain very diverse in comparison with nodal disease. ${ }^{(3,4)}$ However, the heterogeneous manifestation of extranodal DLBCL may infrequently present a diagnostic challenge. A variety of pathologic, flow cytometric and radiologic investigation modalities may need to be employed before a definitive diagnosis can be reached.

We herein describe the case of a 31-year-old man who presented with progressive swelling of the left forearm. He initially opted for herbal remedies, but there was no resolution of symptoms and he subsequently presented to our institution. MR imaging with contrast (coronal D1 DIXON) was performed and showed a large mass involving both the flexor and extensor compartments. Positron emission tomography showed a heterogeneous fluorodeoxyglucose (FDG)-avid left forearm soft tissue mass with a large area of central necrosis (Fig. 1). FDG uptake along the ulna was seen, in keeping with lymphomatous involvement. A biopsy of the forearm mass showed a tumour composed of diffuse sheets of medium to large neoplastic lymphoid cells that were strongly positive for CD20 (Fig. 2a) and CD10 with a Ki-67 level of over 60\%. These findings were consistent with DLBCL (Fig. 2b). Radiologic imaging did not reveal involvement of other sites of the body and the bone marrow was negative for the presence of lymphoma. The patient was treated with six cycles of R-CHOP (rituximab, cyclophosphamide, doxorubicin, vincristine and prednisolone) chemotherapy and radiotherapy, after which he achieved complete remission.

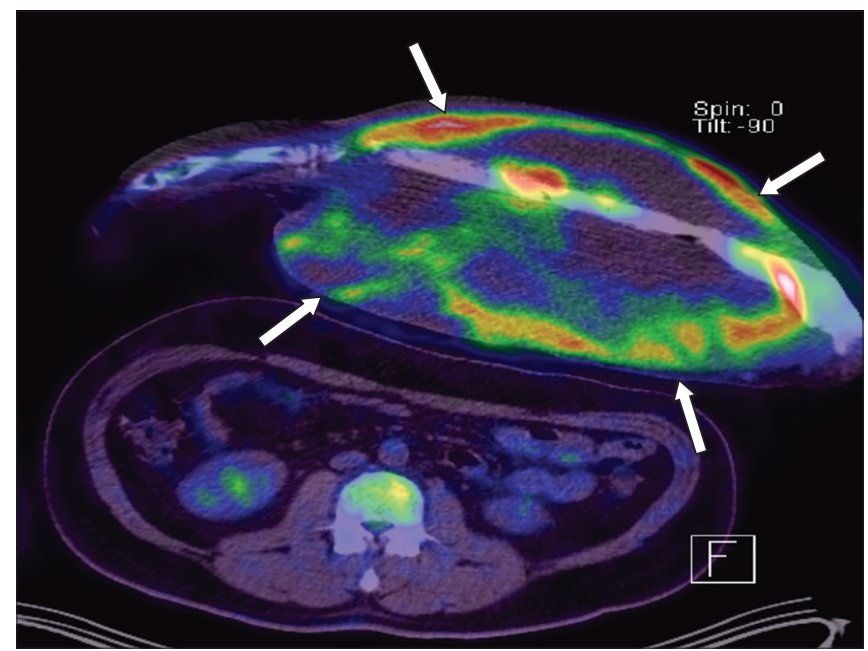

Fig. 1 Fused F-18 fluorodeoxyglucose (FDG) PET-CT image shows a heterogeneous FDG-avid left forearm soft tissue mass (white arrows) with a large area of central necrosis, with FDG uptake along the ulna bone indicating disease involvement.
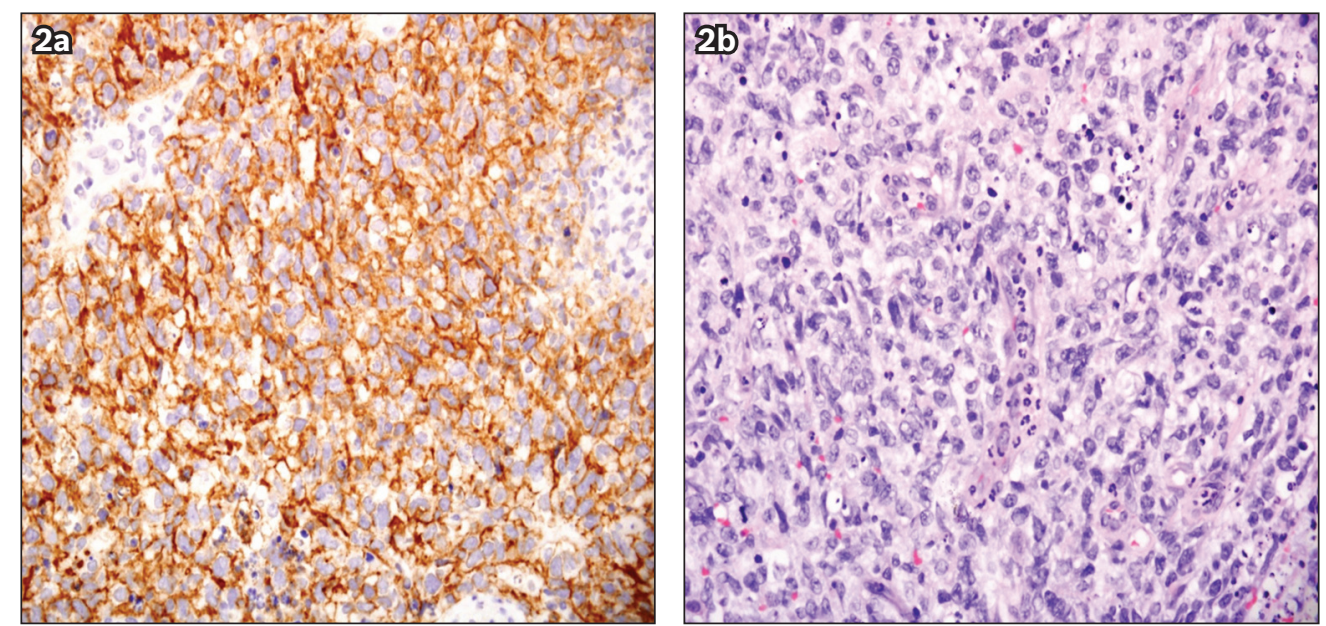

Fig. 2 Photomicrographs show (a) neoplastic lymphoid cells that are positive for CD20 (Haematoxylin \& eosin, x 400); and (b) diffuse sheets of variable medium- to large-sized neoplastic lymphoid cells (Haematoxylin \& eosin, × 4,000). 
Heterogeneous clinical presentations of extranodal DLBCL are well known. Infrequently, they are so varied that the initial clinical impression may not include DLBCL; one such site is at the extremities. Therefore, keeping a high level of suspicion is the key to early diagnosis of DLBCL, which is a highly aggressive but treatable subtype of lymphoma.

Yours sincerely,

Muhammad Bilal $\underline{\mathrm{Abid}}^{1,2}$, Shi $\underline{\mathrm{Wang}}{ }^{3}$, Hoi Yin $\underline{\mathrm{Loi}}^{4}$, Esther Hian Li $\underline{\mathrm{Chan}}^{1}$, Li Mei $\underline{\mathrm{Poon}}^{1}$, Sanjay de Mel ${ }^{1}$

${ }^{1}$ Department of Haematology-Oncology, National University Cancer Institute Singapore, National University Hospital, Singapore, ${ }^{2}$ Division of Internal Medicine, Medical College of Wisconsin, Milwaukee, WI, USA, ${ }^{3}$ Department of Pathology, ${ }^{4}$ Department of Diagnostic Imaging, National University Hospital, Singapore. bilal_abid@hotmail.com

\section{RefERENCES}

1. Smith A, Howell D, Patmore R, Jack A, Roman E. Incidence of haematological malignancy by sub-type: a report from the Haematological Malignancy Research Network. Br J Cancer 2011; 105:1684-92.

2. Campo E, Swerdlow SH, Harris NL, et al. The 2008 WHO classification of lymphoid neoplasms and beyond: evolving concepts and practical applications. Blood $2011 ; 117: 5019-32$

3. Vallangeon BD, Tyer C, Williams B, Lagoo AS. Improved detection of diffuse large B-cell lymphoma by flow cytometric immunophenotyping-Effect of tissue disaggregation method. Cytometry B Clin Cytom 2016; 90:455-61.

4. Abid MB, Nasim F, Anwar K, Pervez S. Diffuse large B cell lymphoma (DLBCL) in Pakistan: an emerging epidemic? Asian Pac J Cancer Prev 2005; 6:531-4. 